\title{
Situação das indústrias madeireiras do estado do Amazonas em 2000.
}

José Ricardo Araújo LIMA'; Joaquim dos SANTOS$^{2}$; Niro HIGUCHI².

\begin{abstract}
RESUMO
Apesar da baixa atividade de usos do solo no Estado, a produção de madeira não é sustentável. Isso é causado, principalmente, pela falta de infra-estrutura dos órgãos responsáveis para monitorar os planos de manejo florestal e de corte raso para agricultura e pecuária. Este estudo apresenta análise de alguns fatores de produção, industrialização e comercialização da indústria madeireira do Estado do Amazonas, visando viabilizar diagnóstico do setor florestal. Essas informações possibilitam o estabelecimento de políticas florestais para a região. Fez-se a definição das zonas de produção, onde os principais municípios produtores de serrados foram Manaus, Itacoatiara, Manacapuru e Parintins; e de compensados e laminados foram Manaus e Itacoatiara. Após essa definição os dados foram coletados diretamente nas indústrias, através de questionários e entrevistas para coletar as seguintes informações: principais espécies consumidas, produtividade das indústrias, origem da matéria-prima, transporte, qualificação de pessoal e treinamento, principais problemas tecnológicos e exportação. $\mathrm{Em} 2000$, a produção estadual foi de $323.400 \mathrm{~m}^{3}$ de madeira em toras. Mais de 50 espécies diferentes são usadas nas serrarias, das quais 16 representaram $80 \%$ do volume total, sendo as 5 espécies (louro inhamui,angelim pedra,amapá,assacu e maçaranduba) contribuíram com 49,34\% do consumo total; quanto as indústrias de compensado e laminado estas usaram, 17 espécies onde as 5 principais (muiratinga, sumaúma, copaíba, assacu e amapá) representaram $62,71 \%$ do consumo total.Também foram coletados dados complementares junto ao IBAMA, SUFRAMA, SEFAZ e IBGE.
\end{abstract}

PALAVRAS-CHAVE

Amazônia, produção madeireira, comercialização.

\section{Lumber industries situation in 2000 in Amazonas state.}

\begin{abstract}
Despite low land activities, hardwood production is not sustainable. This is caused mainly by the lack of infrastructure on the part of the responsible organs that monitor forest management plans and clear cutting for pasture and agriculture. This study dealt with forest industries of the State of Amazonas to better understand the processes of production, industrialization and commercialization, and evaluate their influence on the sustainability of tropical forests. This information is very important in order to establish forest policies for the region. After defining the main forest industry (producers of lumber, plywood and veneer) zones of the State, data were collected directly from the forest industries as well as from IBAMA, SUFRAMA, SEFAZ and IBGE. Manaus, Itacoatiara, Manacapuru and Parintins were the main municipalities of sawn products; and Manaus and Itacoatiara forplywood and veneer. Questionnaires and interviews were used to collect information such as main tree species, transformation productivity, origin of raw material, transportation, personnel qualification and training, and exportation. In 2000, the annual roundwood production in the State of Amazonas was 323,400 $\mathrm{m}^{3}$ (divided equally among sawmill and plywood and veneer industries). More than 50 different tree species are used in the Amazonas sawmills; but only 16 represent $80 \%$ of the total volume. Plywood and veneer industries use 17 tree species. Louro inbamui, angelim pedra, amapá, assacu and maçaranduba are the main species for sawmills; while muiratinga, sumaúma, copaíba, assacu and amapá for plywood and veneer industries.
\end{abstract}

\section{KEYWORDS:}

Amazon, timber prodution, market.

\footnotetext{
${ }^{1}$ Ibama-Instituto Brasileiro do Meio Ambiente e dos Recursos Naturais Renováveis. Rua min. João Gonçalves de Souza, s/no ., BR-319, km 1, CEP: 69075-830, Distrito Industrial-Manaus-AM, Brasil. Tel: +55(92)613-3277, ricardo@santarem.ibama.gov.br.

${ }^{2}$ INPA/CPST - Coordenação de Pesquisas em Silvicultura Tropical, Av. André Araújo 2936, Petrópolis,Caixa Postal 478, CEP:69011-970. Manaus -AM, Brasil. Tel: + 55(0xx92)642-3430,joca@inpa.gov.br; niro@inpa.gov.br
} 


\section{ACTA \\ AMAZONICA}

SITUAÇÃO DAS INDÚSTRIAS MADEIREIRAS

DO ESTADO DO AMAZONAS EM 2000

\section{INTRODUÇÃO}

A Amazônia brasileira legal cobre uma área de, aproximadamente, 5 milhões $\mathrm{km}^{2}$ correspondendo a 2/3 do território nacional. Desse total, mais de 375 milhões hectares são classificados como florestas tropicais densas; descontando os desmatamentos para as mais variadas formas de usos do solo, a área remanescente de floresta primária é de 250 milhões hectares. O estoque de madeira em pé na Amazônia brasileira contribui com mais de 30\% da reserva mundial de madeira dura tropical. Considerando apenas a madeira como mercadoria, isso representa um grande potencial para a economia da região e do Brasil (Almanaque Abril, 2000).

A região amazônica é formada por diferentes tipos florestais. Todavia, do ponto de vista madeireiro, grande parte da matériaprima que abastece o mercado é proveniente de terra-firme exceto o Estado do Amazonas que também é abastecido de matéria-prima oriunda de várzeas.

Atualmente, a região amazônica produz, em torno de 30 milhões de metros cúbicos de madeira tropical em toras por ano, ocupando a terceira posição na produção de madeira tropical, precedido apenas da Malásia e Indonésia (ITTO, 1999). Os Estados do Pará e Mato Grosso são responsáveis por mais de três quartos dessa produção, enquanto que o Amazonas e o Acre detêm produção limitada, com tendência a aumentar sua participação ao longo dos próximos anos (Smeraldi \& Veríssimo, 1999).

O Estado do Amazonas tem uma área de 150 milhões hectares praticamente intactos apenas 2\% desmatados até 1998 (INPE, 2000). As florestas de terra-firme predominam a paisagem do Amazonas, mas, do ponto de vista madeireiro, têm tido uma participação insignificante ao produto interno bruto estadual do setor primário. Além das dificuldades comuns em toda a região amazônica, no Amazonas as questões inerentes à infra-estrutura são potencializadas.

O conhecimento sobre a indústria madeireira amazonense e suas relações com a base florestal é de suma importância para disciplinar o aproveitamento dos recursos estocados, sob o novo paradigma do setor, que é o desenvolvimento sustentável. Isso, aliado ao conhecimento técnico existente, facilitará o entendimento entre o setor produtivo e o Poder Público no cumprimento das normas e leis vigentes. Com isso, as chances de implementar o manejo florestal em regime de rendimento sustentável aumentam consideravelmente, enquanto há cobertura florestal no Estado.

Em termos de madeira, as florestas tropicais, no caso as do Amazonas, representam um potencial enorme para o suprimento de matéria-prima para quaisquer indústrias florestais. Trata-se, entretanto, de um potencial passivo, atualmente sem liquidez, caracterizado pela crescente superexploração e subutilização. $O$ adequado aproveitamento desse potencial pode representar o atendimento da demanda dos países consumidores e riqueza para os países produtores. Caso contrário, as intervenções poderão produzir efeitos negativos catastróficos (Higuchi et al., 1994).

O objetivo deste estudo é produzir um levantamento compreensivo atualizado sobre o setor florestal do Estado do
Amazonas, tendo as seguintes informações: i) consumo anual de madeira em toras pelas serrarias e fábricas de compensado e laminado; ii) número de indústrias madeireiras em operação no Estado do Amazonas; iii) espécies florestais mais consumidas; iv) quantidade e qualidade da mão-de-obra empregada: e v) problemas tecnológicos e legais. Esse levantamento viabilizará um diagnóstico mais realístico do setor florestal, possibilitando o estabelecimento de uma política florestal para o Estado do Amazonas.

\section{MATERIAL E MÉTODOS}

O Estado do Amazonas tem uma área de $1.558 .987,00 \mathrm{~km}^{2}$, correspondendo a $18,42 \%$ do território nacional. Sua localização está entre os paralelos $2^{\circ} 15^{\prime} 30^{\prime \prime}$ de latitude norte e $9^{\circ} 49^{\prime} 13^{\prime \prime}$ de latitude sul e os meridianos 56 $56^{\circ} 05^{\prime} 49^{\prime \prime}$ e $73^{\circ} 48^{\prime} 05^{\prime \prime}$ de longitude oeste de Greenwich, fazendo limite com os estados do Acre, Rondônia, Roraima, Mato Grosso e Pará, tendo ainda uma extensa fronteira de $3.600 \mathrm{~km}$ com os países Venezuela, Colômbia e Peru. Cerca de $61 \%$ de sua superfície fica abaixo dos 100 metros de altitude, 32\% entre 100 e 200 metros e $7 \%$ acima de 200 metros.

Para se conhecer o universo e localização das indústrias madeireiras, foi feito um levantamento junto aos cadastros $\mathrm{e}$ arquivos do IBAMA, onde se definiram principais pólos madeireiros do Estado (serrarias e fábricas de laminado e compensado). Com base nessas informações, selecionou-se as indústrias madeireiras por importância de concentração e por volume de consumo de madeira, onde os principais municípios produtores de serrado foram:Manaus,Itacoatiara, Manacapuru e Parintins, e de laminado e compensado Manaus e Itacoatiara.

Para este trabalho, utilizou-se dados coletados diretamente nas indústrias madeireiras do Estado do Amazonas via entrevistas, com aplicação de um questionário, adaptado de Santos (1986), afim de se obter informações como: principais espécies consumidas, produtividade das indústrias, origem da matéria-prima, transporte, qualificação de pessoal e treinamento, problemas tecnológicos e legais, exportação, bem como informações complementares coletadas em outros Órgãos como IBAMA, SUFRAMA, SEFAZ-AM e IBGE (quantas e quais indústrias estão em funcionamento, quais estão prestando conta de seu volume de madeira comercializado conforme preconiza a legislação). Procurou-se entrevistar o maior número possível de indústrias madeireiras.

\section{RESULTADOS E DISCUSSÕES}

Segundo o Cadastro Geral no Estado do Amazonas do IBAMA, em 2000, 260 serrarias estavam cadastradas no ano de 1999. No entanto, de acordo com os registros da Representação do IBAMA no Estado do Amazonas, somente 39 serrarias estavam funcionando em 1999 e 48 em 2000. Uma das explicações para essa disparidade de dados, dentro do próprio Órgão, é o fato que as indústrias se cadastram no IBAMA e, quando paralisam suas atividades, não dão baixas em seus cadastros. Esse fato é tido como o principal obstáculo para 


\section{ACTA AMAZONICA}

uma avaliação mais criteriosa do setor florestal da Amazônia.

Ao longo dos anos, houve uma oscilação do número de serrarias, com viés de queda. Em 1994, 106 serrarias estavam em funcionamento no Amazonas; em 2000, este número caiu para 48. Os principais fatores têm sido a falta de capital de giro e de incentivo em novas tecnologias (Araújo Lima, 2001).

Entrevistou-se 21 serrarias das 48 registradas no IBAMA e em funcionamento, em 2000, representando 43,8\% da população total (Tabela 1). Foram entrevistadas todas as serrarias consideradas de grande e médio porte e 14 de pequeno porte. Tomando por base o consumo total de madeira apresentado ao IBAMA/AM, em 2000 as 21 serrarias entrevistadas representam $84,57 \%$ do consumo total declarado.

As empresas apontam como um dos fatores responsáveis pela diminuição do número de serrarias, problemas com a legislação ambiental, principalmente, no que diz respeito à legislação estadual, a qual estabelece uma série de exigências, tanto para a indústria, como para os extratores de madeira. Para a extração de madeira, o usuário deve ter em mãos a Autorização de Exploração, expedida pelo IBAMA. O IPAAM (Instituto de Proteção Ambiental do Amazonas) é responsável pelo licenciamento ambiental, quanto às exigências de EPIA/ RIMA (Relatório Prévio de Impacto Ambiental e ou Relatório de Impacto Ambiental) para planos de manejo florestal (áreas superiores a $2.000 \mathrm{ha}$ ).

O consumo anual de madeira em tora, referente ao ano de 2000 , declarado pelas serrarias foi de $136.989,77 \mathrm{~m}^{3}$. Deste consumo, três serrarias de grande porte contribuíram com $70 \%$; quatro serrarias de médio porte com 19\%; e 14 serrarias de pequeno porte, com $11 \%$. As serrarias de pequeno porte entrevistadas consumiram, em média, $1.076,35 \mathrm{~m}^{3}$ de madeira em toras por ano. Dentro deste raciocínio, pode-se estimar que o consumo total, incluindo as 24 pequenas não entrevistadas, foi de $162.822,17 \mathrm{~m}^{3}$.

Comparando, os dados obtidos pela entrevista mais a estimativa do volume das serrarias pequenas não entrevistadas, com os dados declarados ao IBAMA, correspondentes ao ano 2000 , verificou-se que houve uma diferença de $21,6 \%$.

No Estado do Amazonas, 52 espécies florestais são consideradas comerciais e processadas pelas serrarias. No setor industrial madeireiro, uma espécie comercial pode envolver mais de um gênero ou mais de uma espécie botânica. Dessas 52 espécies, 16 representaram 80,44\% do volume total consumido em 2000. Considerando somente as 5 principais espécies (louro inhamui, angelim pedra, amapá, assacu e maçaranduba), observa-se que essas correspondem a 49,34\%, do total deste volume (Tabela 2).

Santos (1988) mostrou que, de um total de 45 espécies mais consumidas em 1985, 11 representavam $83,7 \%$ do consumo total, sendo que somente as 5 principais espécies consumidas naquele ano (jacareúba com 39,5\%, louro com $17,5 \%$, copaíba com $6 \%$, assacu com $5,1 \%$ e saboeiro com $4,2 \%$ ) representavam $72,1 \%$ ( Tabela 3).

Constatou-se que, no ano 2000, as espécies, louro inhamui, Angelim pedra e assacu ainda permanecem como principais espécies consumidas pelas serrarias do Estado do Amazonas, sendo que louro inhamui e assacu ocupam essa posição de destaque, desde 1981 (Santos, 1988). As espécies amapá e maçaranduba que representavam pouco para o consumo, há 8 anos atrás, representaram $15,1 \%$ em 2000.

Observa-se que houve mudanças em relação ao consumo diferenciado das espécies utilizadas pelas serrarias. A espécie louro inhamui se destaca, em todos os anos, com um alto percentual de consumo, enquanto que as espécies, jacareúba, ucuúba, copaíba, muiratinga, caucho, cedro e macacarecuia eram bem representativas nos anos anteriores. Praticamente algumas dessas não são mais consumidas, como é o caso de ucuúba, caucho, cedro, muiratinga e outras com um pequeno percentual, chegando a ser insignificante.

A matéria-prima consumida em 2000, teve 85,1\% de seu volume oriundo de floresta própria e 14,9\% de terceiros, sendo que grande parte dessa madeira é originada de desmatamentos. Em todo o Estado do Amazonas, apenas uma empresa é autosuficiente, ou seja, a origem de sua madeira é de área própria e sob regime de manejo florestal, representando $50 \%$ do consumo total do Estado. A preferência de madeira de origem

Tabela 1 - Distribuição do número de serrarias em funcionamento, pelo porte, em relação ao consumo de matéria-prima, em 2000

\begin{tabular}{ccccc}
\hline \hline \multirow{2}{*}{ ANO } & \multicolumn{5}{c}{ PORTE } \\
\cline { 2 - 5 } & GRANDE & MÉDIO & PEQUENO & TOTAL \\
\hline 2000 & 03 & 04 & 41 & 48 \\
\hline \hline
\end{tabular}

Fonte: Santos, 1986.

Tabela 2 - Consumo das 16 principais espécies utilizadas pelas serrarias do Estado do Amazonas em 2000, em porcentagem.

\begin{tabular}{lc}
\hline \hline Espécies & $\%$ \\
\hline Louro inhamui(Ocotea spp.) & 15,20 \\
Angelim pedra (Dinizia excelsa ) Ducke & 13,40 \\
Amapá (Brosimum spp.) & 9,94 \\
Assacu (Hura crepitans L.) & 5,60 \\
Maçaranduba (Manilkara huberi ) & 5,20 \\
(Ducke)Chevalier & 3,50 \\
Breu (Protium sp.) & 3,37 \\
Sucupira (Andira sp.) & 3,27 \\
Cedrinho (Erisma uncinatum Warm.) & 3,10 \\
Arura Vermelho ( não identificado) & 2,90 \\
Cajui (Anacardium parvifolium.) Ducke & 2,73 \\
Cupiúba (Goupia glabra )Aubl. & 2,73 \\
Uchi Torrado (Endopleura uchi (Huber) Cuatrec.) & 2,57 \\
Jatobá (Hymenaea courbaril L.) & 2,45 \\
Cumaru (Dipteryx odorata (Aubl.) Willd.). & 2,38 \\
\hline Piquia (Caryocar villosum (Aubl.) Pers.) & 19,56 \\
\hline Outras & 100 \\
\hline Total &
\end{tabular}


Tabela 3 - Percentual do volume total produzido pelas serrarias em 1981, 1983, 1985, 1994 e 2000, por espécie.

\begin{tabular}{|c|c|c|c|c|c|}
\hline \multirow{2}{*}{ Espécie } & \multicolumn{5}{|c|}{ Ano } \\
\hline & *1981 & *1983 & *1985 & **1994 & $* * * 2000$ \\
\hline Assacu (Hura crepitans L.) & 0,69 & 3,62 & 5,1 & $\mathrm{x}$ & 5,60 \\
\hline Amapá ( Brosimum spp) & 0 & 0 & $P$ & & 9,93 \\
\hline Andiroba (Carapa guianensis Aubl.) & 1,04 & 1,63 & & $\mathrm{x}$ & 0,14 \\
\hline Angelim pedra (Dinizia excelsa) Ducke & 1,53 & 1,67 & & $\mathrm{x}$ & 13,34 \\
\hline Arura Vermelho (não identificado) & 0 & 0 & 0 & & 3,10 \\
\hline Breu (Protium sp.) & 0 & 0 & 0 & & 3,44 \\
\hline Cajui (Anacardium parvifolium) Ducke & 0 & 0,03 & $\mathrm{P}$ & & 2,87 \\
\hline Caucho (Castilla ulei Warb.) & 3,41 & 3,62 & 3,6 & & 0 \\
\hline Cedrinho (Erisma uncinatum Warm) & 0 & 0 & & $\mathrm{x}$ & 3,26 \\
\hline Cedro (Cedrela odorata) L. & 2,85 & 0,67 & 1,7 & $x$ & 0 \\
\hline Copaíba (Copaifera multijuga) Haine & 7,6 & 7,92 & 6,0 & $x$ & 0,07 \\
\hline Cumaru (Dipterix odorata (Aubl) Willd.) & 0 & 0 & & & 2,46 \\
\hline Cupiúba (Goupia glabra) Aubl. & 0,04 & 0,06 & $P$ & $\mathrm{x}$ & 2,74 \\
\hline Jacareúba (Calophyllum angulare)A.C. & 26,40 & 35,62 & 39,5 & $\mathrm{x}$ & 1.66 \\
\hline Jatobá (Hymenaea courbaril L.) & 0 & 0 & 0 & & 2,58 \\
\hline Louro inhamui (Ocotea sp) & 21,21 & 27,32 & 17,5 & $\mathrm{x}$ & 15,15 \\
\hline Macacarecuia (Eschweilera albiflora)(DC)Miers & 2,01 & 5,11 & 3,2 & & 0,07 \\
\hline Maçaranduba (Manilkara huberi (Ducke)Chevalier)) & 0,01 & 0 & $\mathrm{P}$ & & 5,15 \\
\hline Mata-Mata Eschweilera coriacea (DC.)S.A.Mori. & 0 & 0 & 0 & & 1,90 \\
\hline Muiratinga (Maquira coriacea (H.Karst.)C.C.Berg & 5,75 & 1,14 & 0,4 & & 0 \\
\hline Piquiá (Cariocar villosum (Aubl.)Pers.) & 0 & 0 & $P$ & $\mathrm{x}$ & 2,38 \\
\hline Sucupira (Andira sp) & 2,28 & 1,71 & $\mathrm{P}$ & $\mathrm{x}$ & 3,38 \\
\hline Uchi Torrado (Endopleura uchi (Huber)Cuatrec. & 0 & 0 & 0 & & 2,73 \\
\hline Ucuúba (Virola surinamensis ) Rol.ex Rottb.)Warb. & 21,94 & 4,37 & 2,5 & & 0 \\
\hline $\begin{array}{l}\text { Saboeiro (Zygia racemosa) (Ducke)Barneby } \\
\text { J.W.Grines }\end{array}$ & 0 & 0 & 4,2 & & 0 \\
\hline TOTAL & 96,76 & 94,49 & 83,7 & & 81,95 \\
\hline
\end{tabular}

Fonte:

(*) Santos 1988; (**) Hummel 1994 o autor não apresentou o percentual, o símbolo "x" representa que a espécie era uma das principais utilizadas no Estado;

(***) Aplicação Questionário 2000; P - Espécies muito pouco consumidas

de desmatamentos se dá, principalmente, pela falta de recursos financeiros para garantir os custos de elaboração e execução de projetos de planos de manejo florestal.

Do total de matéria-prima (toras) recebida pelas 21 serrarias entrevistadas, 96,85\% tem procedência de terra-firme. Apesar desse alto percentual de origem das toras, o tipo de transporte utilizado não é apenas terrestre, aparecendo com freqüência $o$ fluvial, principalmente, através de balsas e jangadas.

Em 2000, somente 6 fábricas de compensado e laminado estavam em funcionamento, sendo 4 no município de Manaus e 2 em Itacoatiara. Dessas, 5 foram entrevistadas, as quais representam 90,32\% do consumo declarado ao IBAMA.

Os dados obtidos, entrevistando 5 indústrias, mostram queo consumode madeira em toras, em 2000 , foide $146.586 \mathrm{~m}^{3}$. Somente uma indústria é responsável por $55,23 \%$ deste consumo. Na tabela 4 encontram-se os volumes relativos ao consumo declarado ao IBAMA referentes a cada indústria neste mesmo ano. Assumindo que a indústria não entrevistada representa 9,60\% do consumo total declarado ao IBAMA, $\left(13.969 \mathrm{~m}^{3}\right)$, pode-se afirmar que o consumo de madeira em toras pela indústria de compensado e laminado do Estado do Amazonas em 2000, foi de $160.555 \mathrm{~m}^{3}$.

Ao comparar os dados obtidos através de entrevista nas 5 indústrias, com os apresentados ao IBAMA, nota-se que há uma diferença em torno de $10,50 \%$ no consumo total de madeira em toras.

As indústrias de compensado e laminado, no ano de 2000,utilizaram 17 espécies florestais, estas representando $82,22 \%$ do total consumido (Tabela 5); sendo que somente as 5 principais contribuíram com $62,71 \%$ do total.

Considerando os anos de 1981, 1983, 1985, 1994 e 2000, observa-se que as indústrias de compensado e laminado também apresentaram mudanças, em relação ao consumo das principais espécies utilizadas, (Tabela 6). Nota-se que copaíba e muiratinga apresentam alto percentual de consumo e praticamente 


\section{ACTA AMAZONICA}

constante ao longo de todos os anos. Quanto a ucuúba, espécie com alto percentual de consumo nos anos anteriores, não teve participação no consumo das indústrias no ano 2000 , fato este atribuído, principalmente, pela redução do número de indivíduos com DAP superior a $40 \mathrm{~cm}$, em conseqüência da exploração seletiva ocorrida durante os últimos anos. Ainda pode-se observar que somente muiratinga, sumaúma e copaíba representam mais de $51 \%$ do consumo.

A origem da matéria-prima para a indústria de compensado e laminado, no ano 2000 , foi de floresta de terceiros $(69,80 \%)$ e de floresta própria $(30,20 \%)$. Em relação ao tipo florestal, $74,58 \%$ da matéria-prima é de várzea e $25,42 \%$ de terra-firme.

Quanto ao transporte utilizado até a indústria, o principal é o fluvial (através de jangadas e com menor freqüência balsa) .

Com base nos dados obtidos pela entrevista, tem-se que o consumo estimado da madeira em toras para as serrarias do Estado foi de $162.822,17 \mathrm{~m}^{3}$, com aproveitamento médio de $27 \%$, logo, estima-se que a produção desse segmento de indústria madeireira foi em torno de $44.000 \mathrm{~m}^{3}$.

Quanto às fábricas de compensado e laminado, o consumo de madeira em toras foi semelhante ao das serrarias, $160.555 \mathrm{~m}^{3}$, sendo que o aproveitamento médio declarado foi de $50 \%$, fazendo com que a produção deste segmento alcançasse, aproximadamente, $80.300 \mathrm{~m}^{3}$. Somando-se a produção destes dois segmentos tem-se que a produção da indústria madeireira do Estado do Amazonas, em 2000, foi de $124.300 \mathrm{~m}^{3}$ de madeira beneficiada.

Com relação aos dados obtidos pela entrevista, tem-se que o consumo de madeira em toras foi $15,7 \%$ superior ao declarado ao IBAMA, para este período, demonstrando que a variação existente entre fontes de informações dificulta o diagnóstico sobre o setor, pelo fato da não confiabilidade nos dados existentes.

A produção de madeira das 14 maiores indústrias representa em torno de $74 \%$ (1998), 86\% (1999) e $82 \%$ (2000), dovolume total produzido pelo Estado (Tabela 7), considerando-se dados doIBAMA.

A tabela 8 apresenta dados coletados junto ao IBAMA/ AM,sobre volume exportado para o mercado internacional, de produtos madeireiros serrados, compensados e laminados, durante o período de 1998-2000. Com base nas estatísticas da ITTO (até 1995), a contribuição do Amazonas ao mercado internacional de madeira dura tropical é de, aproximadamente $0,2 \%$, enquanto que a participação do Estado no volume total exportado pela região amazônica estaria em torno de 3\%. Apesar de insignificante participação no mercado madeireiro, nos anos 1998, 1999 e 2000, as exportações de madeira corresponderam a 15,5\%, 7,6\% e 3,1\%, respectivamente, do total das exportações do distrito industrial da Zona Franca de Manaus (A Crítica,2001).

A exportação dos produtos serrados tem aumentado nos últimos anos, coincidindo com a diminuição das exportações realizadas pelos principais exportadores do Sudeste asiático como Malásia e Indonésia (Higuchi, 1997). No Amazonas, 11 empresas de madeira serrada exportaram produtos para o exterior em 2000, sendo que apenas 2 dessas contribuíram com $88 \%$ do volume total exportado. São 9 países importadores da madeira serrada do Estado e os principais
Tabela 4 - Consumo de madeira em toras pela indústria de compensado e laminado declarado ao IBAMA, em 2000.

\begin{tabular}{cc}
\hline \hline Empresa & Volume $\left(\mathrm{m}^{3}\right)$ \\
\hline A & 46.410 \\
B & 32.173 \\
C & 25.489 \\
D & 13.969 \\
E & 13.687 \\
F & 13.317 \\
\hline Total & 145.045 \\
\hline \hline
\end{tabular}

Fonte: www.ibama.gov.br

Tabela 5 - Percentual do consumo das espécies utilizadas pela Indústria de compensado e laminado do Estado do Amazonas em 2000.

\begin{tabular}{lc}
\hline \hline Espécie & $\%$ \\
\hline Muiratinga (Maquira coriacea & 19,71 \\
(H.Karst.)C.C.Berg & 17,18 \\
Sumaúma (Ceiba pentandra (L.) Gaertn.) & 14,7 \\
Copaíba (Copaifera multijuga)Haine & 7,32 \\
Assacu (Hura crepitans L.) & 3,80 \\
Amapá (Brosimum spp) & 3,01 \\
Cedrinho (Erisma uncinatum Warm.) & 2,73 \\
Garrote (Brosimum utile (Kunth)Oken ex J.Presl & 2,38 \\
Guariúba (Clarisia racemosa Ruiz. \& Pav.) & 2,15 \\
Cajuí (Anacardium spp.) & 1,79 \\
Breu (Protium sp) & 1,75 \\
Xuru (não identificado) & 1,38 \\
Paricá (Virola theiodora (Spruce ex & 1,19 \\
Benth.)Warb.) & 1,15 \\
Paricarana (Parkia spp) & 1,02 \\
Tauari (Allantoma lineata)(Mart.O.Berg.)Miers & 0,75 \\
Macaca (Platymiscium duckei Huber) & 0,21 \\
Virola (Virola surinamensis) (Rol.exRottb.)Warb. & 17,78 \\
\hline Axixá (Sterculia excelsa Mart.) & 100,00 \\
\hline Outras & \\
\hline Total &
\end{tabular}

são Estados Unidos, Portugal, Alemanha e Holanda, que juntos importam mais de $80 \%$ do volume total.

A exportação de compensados durante o período 19982000 foi sempre superior a de laminados, acentuando a diferença em 2000, quando a exportação de laminados foi inferior a $10 \%$ da exportação de compensados. Uma causa provável para explicar essa diminuição foi o fechamento de uma fábrica, em 2000. Além disso, o abastecimento de toras para as fábricas de compensados e laminados foi prejudicado pela sazonalidade local, que dificultou a extração de madeira nas várzeas. Em 2000, 2 empresas foram responsáveis por $80 \%$ da exportação de compensados e laminados realizada pelas indústrias amazonenses. São 16 países importadores 
Tabela 6 - Percentual do volume total produzido das principais espécies consumidas pela indústria de compensado e laminado em 1981, 1983, 1985, 1994 e 2000, em porcentagem.

\begin{tabular}{|c|c|c|c|c|c|}
\hline Espécie & *1981 & *1983 & *1985 & **1994 & ***2000 \\
\hline Assacu (Hura crepitans L.) & $\mathrm{P}$ & $\mathrm{p}$ & $\mathrm{P}$ & $\mathrm{X}$ & 7,32 \\
\hline Amapá (Brosimum sp) & $P$ & $P$ & $P$ & $X$ & 3,80 \\
\hline Caucho (Castilla ulei Warb.) & 9,70 & 8,20 & 5,80 & $\mathrm{X}$ & 0,00 \\
\hline Copaíba (Copaifera multijuga Haine) & 27,50 & 15,80 & 20,00 & $\mathrm{X}$ & 14,70 \\
\hline $\begin{array}{l}\text { Jacareúba (Calophyllum angulare } \\
\text { A.C.Sm.) }\end{array}$ & 3,00 & 6,70 & 2,50 & & 0,00 \\
\hline $\begin{array}{l}\text { Muiratinga (Maquira coriacea } \\
\text { (H.Karst.)C.C.Berg) }\end{array}$ & 19,30 & 20,90 & 19,20 & & 19,71 \\
\hline Sumaúma (Ceiba pentandra (L.)Gaertn.) & 1,50 & 18,40 & 32,70 & $x$ & 17,18 \\
\hline $\begin{array}{l}\text { Ucuúba (Virola surinamensis (Rol.ex } \\
\text { Rottb.)Warb.) }\end{array}$ & 38,00 & 14,40 & 12,10 & & 0,00 \\
\hline TOTAL & 99,00 & 84,40 & 92,30 & & 62,70 \\
\hline
\end{tabular}

Tabela 7 - Produção e exportação de madeira das 14 maiores indústrias, 1998, 1999 e 2000 (m³) do Estado do Amazonas.

\begin{tabular}{|c|c|c|c|}
\hline & INSTITUIÇÃO & *PRODUÇÃO & **EXPORTAÇÃO \\
\hline \multirow{2}{*}{1998} & SUFRAMA & 119.763 & 78.203 \\
\hline & IBAMA & 119.811 & 75.711 \\
\hline \multirow{2}{*}{1999} & SUFRAMA & 130.226 & 39.787 \\
\hline & IBAMA & 124.339 & 61.168 \\
\hline \multirow{2}{*}{2000} & SUFRAMA & 91.157 & 48.562 \\
\hline & IBAMA & 112.719 & 51.207 \\
\hline
\end{tabular}

Fonte: www.gov.br; SUFRAMA (2000).

(*) refere-se ao somatório da produção de madeira serrada, compensado e laminado; (**) refere-se ao somatório da exportação de madeira serrada, compensado e laminado.

de compensados e laminados do Estado do Amazonas, sendo os principais: Estados Unidos, Reino Unido e Alemanha, contribuindo, respectivamente com 35\%, $30 \%$ e $10 \%$ do volume total exportado. Santos \& Hummel (1988) identificaram que no ano de 1986 os Estados Unidos e o Reino Unido já eram os principais parceiros na importação de madeira (serrado e compensado) do Estado do Amazonas.

\section{CONCLUSÕES}

Do total produzido em 2000, equivalente em toras, aproximadamente, $50 \%$ foram exportados em forma de serrados, compensados e laminados. Analisando os anos de 1998, 1999 e 2000, as exportações de serrados vêm gradualmente crescendo, enquanto que, compensados e laminados vêm diminuindo.

$\mathrm{O}$ aproveitamento médio das toras transformadas nas serrarias, de acordo com os entrevistados, foi de $27 \%$; o restante, $73 \%$, é resíduo que, na maioria das vezes, é queimado a céu aberto. Nas fábricas de compensado e laminado, o aproveitamento é um pouco maior do que nas serrarias, alcançando $50 \%$.
Dentre as 48 serrarias existentes em 2000, 3 são de grande porte e são responsáveis por $70 \%$ do consumo total da madeira em toras do Estado e 4 são serrarias de médio porte que contribuem com outros 19\%. Entre as indústrias de compensado e laminado, apenas duas empresas contribuíram com mais de $75 \%$ do total produzido no ano de 2000.

Das mais de 50 espécies comerciais (geralmente morfoespécies) utilizadas pelas serrarias do Estado do Amazonas, somente 16 representam $80,44 \%$ do volume total consumido, sendo que as espécies louro inhamui, angelim pedra, amapá, assacu e maçaranduba, contribuíram com 49,34\% deste volume.

Nas serrarias, o louro inhamui se destaca com alto percentual de consumo. As espécies ucuúba, caucho, cedro, muiratinga, jacareúba,copaíba e macacarecuia eram bem representativas nos anos anteriores, sendo que em 2000, as 4 primeiras citadas, praticamente, desapareceram do mercado, possivelmente, pela concorrência com as fábricas de laminado e compensado.

Em 2000, as indústrias de compensado e laminado utilizaram 17 espécies, das quais apenas 5 espécies contribuíram com $62,71 \%$ do consumo total. Essas espécies são: muiratinga, sumaúma copaíba assacu e amapá. sumaúma, muiratinga e 


\section{ACTA AMAZONICA}

Tabela 8 - Produtos madeireiros exportados pelo Estado do Amazonas, no período 1998-2000 $\left(\mathrm{m}^{3}\right)$.

\begin{tabular}{|c|c|c|c|}
\hline \multirow{2}{*}{ Produtos } & \multicolumn{3}{|c|}{ Anos } \\
\hline & 1998 & 1999 & 2000 \\
\hline 1) Serrados & 7.851 & 13.454 & 14.675 \\
\hline 2) Compensados & 36.939 & 29.714 & 33.493 \\
\hline 3) Laminados & 30.920 & 17.863 & 3.037 \\
\hline 4) $2+3$ & 67.859 & 47.577 & 36.530 \\
\hline Exportação total & 75.710 & 61.031 & 51.205 \\
\hline
\end{tabular}

Fonte: www.gov.br

copaíba são as espécies mais utilizadas no consumo das fábricas desde 1983, representando acima de $50 \%$ do consumo total.

Em se tratando de serrarias e levando-se em consideração o comércio de matéria-prima, apenas uma empresa é autosuficiente, ou seja, a origem de sua madeira é de área própria e sob regime de manejo florestal. É pertinente salientar que essa serraria é responsável por $85 \%$ do abastecimento total de matéria-prima. Quanto às fábricas de laminado e compensado, atualmente nenhuma é auto suficiente no que diz respeito a abastecimento de matéria prima. Constatou-se que $70 \%$ do abastecimento é oriundo de outras propriedades.

Um dos principais problemas indicado pelos donos das indústrias madeireiras do Amazonas é o rigor da aplicação da legislação ambiental, principalmente, no que diz respeito à legislação estadual, a qual estabelece uma série de exigências, tanto para a indústria, como também para os extratores de madeira, burocratizando a concessão de qualquer instrumento de legalização da atividade florestal e/ou industrial.

Há uma percepção da inconsistência e disparidade dos dados disponíveis entre as Instituições, bem como dentro de algumas que são responsáveis pela estatística, tornando tais dados, não confiáveis. Por causa das variações existentes entre fontes de informação, o diagnóstico sobre o setor é dificultado. O principal fator que torna tais dados não confiáveis é o conflito entre as legislações vigentes; algumas incentivam a superestimativa da produção para atrair investimentos mais altos, enquanto outras, ao contrário, incentivam a sub-estimativa para diminuir o pagamento de taxas e impostos.

A falta de integração entre as instituições, principalmente, de fiscalização e fomento, em relação a compatibilização dos bancos de dados propicia incorreções. Essas diferenças trazem grandes prejuízos à arrecadação estadual, dando, ao mesmo tempo, insegurança quanto ao planejamento e organização dos Órgãos envolvidos com a questão madeireira, aliada à precariedade dos instrumentos sobre o controle e monitoramento da atividade madeireira no Estado. O ideal é cruzar todos os bancos de dados para unificá-los e centralizálos em um único órgão do Poder Público.

Na questão do manejo florestal constante no Decreto $\mathrm{n}^{\mathrm{o}}$ 2.788 de 28/09/98, os dados governamentais disponíveis, infelizmente, não podem ser usados para diagnosticar essa prática. A realidade é que, em muitos planos, seus executores não cumprem o que vem descrito no projeto sobre as práticas e técnicas necessárias à condução correta desses projetos, proporcionando informações distorcidas e incompletas. Ainda que a produção do Amazonas seja insignificante em relação à produção da região amazônica, essa falta de avaliação pode comprometer a sustentabilidade da produção das florestas do Estado.

A madeira de origem de desmatamentos, autorizados ou não, é a forma de mais fácil acesso, sendo um fator principal para o desprezo dos consumidores de madeira quanto à prática do manejo florestal sustentável. Atrelado a isso, há a ineficiência dos instrumentos de controle para detectar a atividade ilegal, que favorece a extração predatória de madeira, diminuindo a competitividade dos produtos de planos de manejo florestal sustentável. Se for difícil criar incentivos para a prática do manejo florestal sustentável, a alternativa é criar desincentivos para a extração predatória legal ou ilegal.

\section{BIBLIOGRAFIA CITADA}

A Crítica. 2001. Suframa - Exportação da Zona Franca. Jornal A Crítica. Dia. 25-26, Fevereiro. Manaus-AM.

Araújo Lima, J.R. 2001. Indústria Madeireira no Amazonas: Caracterização, Produção e Comercialização. Dissertação de mestrado.INPA/UA, Manaus-AM. 62pp.

Higuchi, N. 1997. A exploração seletiva de madeira na Amazônia brasileira: sua relação com o desmatamento e o mercado internacional de madeira dura tropical. In: BIONTE. p. 1430. Relatório Final.

Higuchi, N.; Hummel, A.C.; Freitas, J.V. de; Malinovski, J.R.; Stokes, B.J. 1994. Exploração florestal nas várzeas do Estado do Amazonas: seleção de árvores, derrubada e transporte. In: Seminário de Atualização sobre Sistemas de Colbeita de Madeira e Transporte Florestal. Curitiba, p. 168-193.

Hummel, A. C. 1994. Diagnóstico do Subsetor Madeireiro do Estado do Amazonas. Série Estudos Setoriais. Edição SEBRAE, Manaus. 73pp.

INPE. 2000. Desflorestamento na Amazônia Brasileira. São José dos Campos. 15pp. (Mimeo.)

ITTO (International Tropical Timber Organization) , 1999. Production and Consumption. Annual Review And Assessment Of The World Tropical Timber Situation 1998. (www.itto.or.ip/timbersituation/timber1998/prodution.html).

Pandolfo, C. 1979. A Amazônia brasileira e suas potencialidades. Belém: SUDAM. 73pp.

Santos, J. dos 1986. Situação da indústria madeireira no município de Manaus (1981 -1983) e das serrarias do Estado do Amazonas. Dissertação de Mestrado. Universidade Federal do Paraná. Curitiba, Paraná. 78pp.

Santos, J. dos 1988. Diagnóstico das serrarias e das fábricas de laminados e compensados do Estado do Amazonas. Acta Amazonica, 18 (1-2):67-82. 
Santos, J. dos; Hummel, A.C. 1988. Situação das exportações de madeira serrada, laminada e compensada do estado do Amazonas 1984,1985 e 1986, In: Encontro Brasileiro de Economia Florestal, v.2. Curitiba: EMBRAPA/CPNF. p.415-29.

Smeraldi, R; Veríssimo, A. 1999. Acertando o Alvo: consumo de madeira no mercado interno brasileiro e promoção da certificação florestal. FOE, IMAFLORA \& IMAZON. 41pp.
SUFRAMA. 2000. Sistemas Indicadores Industriais - Pólo Madeireiro. (www.ibama.gov.br)

RECEBIDO EM 26/03/2003

ACEITO EM 19/01/2005 